\title{
Optimizing the operating conditions in a high precision industrial process using soft computing techniques
}

\section{Emilio Corchado ${ }^{1}$, Javier Sedano ${ }^{2}$, Leticia Curiel $^{3}$ and José R. Villar ${ }^{4}$}

(1) Departamento de Informática y Automática, Universidad de Salamanca, Campus Plaza de la Merced s/n, 37008 Salamanca, Spain

Email: escorchado@usal.es

(2) Department of Artificial Intelligence and Applied Electronics, Technological Institute of Castilla and Leon, Poligono Industrial de Villalonquejar, C/Lopez Bravo 70, 09001 Burgos, Spain

(3) Department of Civil Engineering, University of Burgos, EPS Politécnica, Campus Vena, Edificio C. C/Francisco de Vitoria, s/n, 09001 Burgos, Spain

(4) Department of Computer Science, University of Oviedo, Campus de Viesques s/n, 33204 Gijón, Spain

\begin{abstract}
This interdisciplinary research is based on the application of unsupervized connectionist architectures in conjunction with modelling systems and on the determining of the optimal operating conditions of a new high precision industrial process known as laser milling. Laser milling is a relatively new micro-manufacturing technique in the production of high-value industrial components. The industrial problem is defined by a data set relayed through standard sensors situated on a laser-milling centre, which is a machine tool for manufacturing high-value micro-moulds, micro-dies and micro-tools. The new three-phase industrial system presented in this study is capable of identifying a model for the laser-milling process based on low-order models. The first two steps are based on the use of unsupervized connectionist models. The first step involves the analysis of the data sets that define each case study to identify if they are informative enough or if the experiments have to be performed again. In the second step, a feature selection phase is performed to determine the main variables to be processed in the third step. In this last step, the results of the study provide a model for a laser-milling procedure based on low-order models, such as black-box, in order to approximate the optimal form of the laser-milling process. The three-step model has been tested with real data obtained for three different materials: aluminium, copper and hardened steel. These three materials are used in the manufacture of micro-moulds, micro-coolers and micro-dies, high-value tools for the medical and automotive industries among others. As the model inputs are standard data provided by the laser-milling centre, the industrial implementation of the model is immediate. Thus, this study demonstrates how a high precision industrial process can be improved using a combination of artificial intelligence and identification techniques.
\end{abstract}

Keywords: unsupervized learning, exploratory projection pursuit, modelling systems, industrial applications 


\section{Introduction}

Owing to the fast development of the manufacturing capabilities of countries such as China and India, traditional manufacturing is currently looking for new challenges. One such opportunity is in the manufacture of high-value micro-tools for different industrial sectors. Examples of these tools are those used in the field of medical therapeutics (odonto-stomatology) for bucco-dental rehabilitation and restoration in the processing and manufacturing of buccodental prosthesis, such as partial crowns, inlays and onlays, and partial and complete prosthesis fitted on structures of different metals, such as titanium, chrome cobalt, noble metals, etc., in which the optimizing of the registering and mapping of the surgical field to be operated on is required. Other examples are steel moulds with deep marking for serial numbers or barcodes for quality control for the automotive industry, aluminium moulds with highly complex 3D micro-shapes for medical applications or copper electrodes for electrical discharge machining (EDM). These tools are characterized by requiring critical $3 \mathrm{D}$ shapes or deep vertical walls somewhere on their surfaces. The generation of these geometries can be done using high-accuracy, high-speed milling or EDM, but both technologies have a physical limit where the $3 \mathrm{D}$ shapes are very small. A new technology is called for to surpass this limit: laser milling (Ion, 2005).

Laser milling consists in the controlled evaporation of material caused by its interaction with a high-energy pulsed laser beam. The amount of vaporized material depends not only on laser pulse characteristics, but also on the composition of the material to be removed (Kuhl, 2002; Henry et al., 2004). A conventional milling machine knows in every moment the amount of material removed (the whole volume of the mill), but this is not so easy for a lasermilling centre. The usual proposal to solve this problem is the development of analytical or empirical models fit to the process behaviour (Harrison et al., 2004; Tani et al., 2008; Witte et al., 2008). However, these models always take those variables that perfectly fit the physical process as input data. Unfortunately, these variables cannot be measured easily on realworld industrial machines that implement lasermilling technology. Therefore, to facilitate the quick take-up of this technology by industry, it is necessary to develop a high precision model that can predict the exact amount of material that each laser pulse can get out using input data variables that can be obtained directly from real-world machines. This model will provide the control of laser milling with the accuracy required for micro-tools and, also, the optimization of their manufacture. In this interdisciplinary study, such a model is obtained using a combination of conventional and soft computing models. Soft computing (Cruz \& Pelta, 2009; Corchado et al., 2010; Torra \& Narukawa, 2010) is a collection or set of computational techniques in machine learning (Abraham et al., 2009), such as artificial neural networks (Kohonen, 2006; Herrero et al., 2010), genetic algorithms (Lorena \& de Carvalho, 2008; Naldi et al., 2008), fuzzy systems (Zadeh, 2009; Berlanga et al., 2010) and swarm intelligence (Das et al., 2008), which investigate, simulate and analyse very complex issues and phenomena.

Unsupervized learning is used initially, as a preliminary phase before the modelling system is established, to analyse the internal structure of the data sets. Consequently, it is worth knowing whether the data sets are relevant and informative enough. Exploratory projection pursuit (EPP) (Diaconis \& Freedman, 1984; Caló, 2007) is a statistical method aimed at solving the difficult problem of identifying structures in high-dimensional data, providing an interesting view of the internal structure of the data set representing the problem to be analysed using higher-order statistics such as kurtosis, which is a measure of how pointed a distribution is.

In EPP, a relevant structure is usually defined with respect to the fact that most projections of high-dimensional data onto arbitrary lines through most multi-dimensional data give almost Gaussian distributions (Diaconis \& Freedman, 1984). Thus, interestingness is usually 
defined in terms of how far the distribution is from the Gaussian distribution.

These models are also used in a second step to carry out feature selection (Guyon \& Elisseeff, 2003; Liu \& $\mathrm{Yu}, 2005)$ to identify the main variables to be used in the third step. Several neural projection models based on EPP are applied in this study to carry out the first two steps of this soft computing model.

In complex, multi-dimensional domains, such as in industry, some data sets may hinder their own internal structures. Variables may contain false correlations that hinder the process of detecting the underlying causes of a data set. Furthermore, some features may be redundant because the information they add is contained in other features or variables. Extra features may increase computation time and can interfere in the accuracy of the clustering or classification process.

Feature selection (Guyon \& Elisseeff, 2003; Liu \& Yu, 2005) improves classification by searching for the subset of features that best classifies the training data and decreasing computation time.

Finally, the third and last phase is based on the use of classical identification techniques to obtain a model of the normal operating conditions.

Thus, unsupervized learning, and specifically EPP, is used in conjunction with classical identification techniques to obtain a model of the dynamics for a real-world industrial process, laser milling in this case. EPP is used to extract the relevant structures and relationships between variables to guarantee that the data set obtained by the sensors during the experiments is informative enough to identify the most significant features. The classical identification techniques then model the laser-milling conditions to choose the correct working parameters. Finally, the estimated working parameters facilitate increasing the quality of the resulting pieces.

This study presents the three-step procedure designed to identify the optimal conditions of a laser-milling process. The paper is organized as follows: Section 2 introduces the unsupervized connectionist techniques used for analysing the data to extract the relevant internal structures. This is the first step in the modelling process. The second step, feature selection, is described in Section 3, which serves to select the main variables to be processed in the third step. Section 4 describes the classical identification techniques used in the system modelling (third step). Section 5 provides details on the application field and the case studies and an analysis and comparison of the best models and results. Lastly, conclusions and future work are discussed.

\section{Relevant internal structure extraction using projection methods}

Principal component analysis (PCA) (Esbensen $\&$ Geladi, 2009), first found in Pearson's research (Pearson, 1901) and independently in Hotelling's (Hotelling, 1933), is a statistical method describing multivariate data set variations as uncorrelated variables, each of which is a linear combination of the original variables. Its main goal is to derive new variables in decreasing order of importance (variance), which are linear combinations of the original variables and are uncorrelated with each other. It is a well-known technique that can be implemented by a number of connectionist models (Oja, 1982; Fyfe, 1993). The PCA aims to find that orthogonal basis that maximizes the data's variance for a given dimensionality of basis. The PCA is the most frequently reported linear operation involving unsupervized learning for data compression and feature selection.

The standard statistical method of EPP (Friedman \& Tukey, 1974; Corchado et al., 2004; Caló, 2007) provides a linear projection of a data set. The data projections make use of a set of basis vectors that best reveals the relevant structures of the data. The relevancy is measured as interestingness, which is usually defined in terms of how far the distribution is from the Gaussian distribution (Seung et al., 1998).

One neural implementation of EPP is maximum likelihood Hebbian learning (MLHL) 
(Corchado et al., 2004). MLHL has been widely used in the field of pattern recognition (Corchado \& Fyfe, 2003; Corchado et al., 2004) as an extension of PCA. It identifies interestingness (Friedman \& Tukey, 1974; Corchado et al., 2004) by maximizing the probability of the residuals under specific probability density functions that are non-Gaussian under the analysis of the fourth-order statistic, the kurtosis.

An extended version of this model is the cooperative maximum likelihood Hebbian learning (CMLHL) (Corchado \& Fyfe, 2003). CMLHL is based on MLHL with the addition of lateral connections (Corchado \& Fyfe, 2003) derived from the rectified Gaussian distribution (Seung et al., 1998). The resulting network can find the independent factors of a data set but it does so in a way that captures some type of global ordering in the data set.

Consider an $N$-dimensional input vector $(\boldsymbol{x})$, an $M$-dimensional output vector $(y)$ and a weight matrix $\boldsymbol{W}$, where the element $W_{i j}$ represents the relationship between input $x_{j}$ and output $y_{i}$. Then, as shown in Corchado and Fyfe (2003), the CMLHL can be carried out as a four steps procedure as follows:

Feed-forward step $\quad y_{i}=\sum_{j=1}^{\mathrm{N}} W_{i j} x_{j}, \forall i$

Lateral activation passing

$$
y_{i}(t+1)=\left[y_{i}(t)+\tau(b-A y)\right]^{+}
$$

Feedback step $\quad e_{j}=x_{j}-\sum_{i=1}^{M} W_{i j} y_{i}, \forall j$

Weights changing step

$$
\Delta W_{i j}=\eta y_{j} \operatorname{sign}\left(e_{j}\right)\left|e_{j}\right|^{p-1}
$$

where $\eta$ is the learning rate, the rectification [ $]^{+}$ is necessary to ensure that the $y$-values remain within the positive quadrant; $\tau$ is the 'strength' of the lateral connections, $b$ the bias parameter and $p$ is a parameter related to the energy function (Corchado \& Fyfe, 2003; Corchado et al., 2004).
A is a symmetric matrix used to modify the response to the data whose effect is based on the relation between the distances among the output neurons. It is based on the cooperative distribution, but to speed learning up, it can be simplified to

$$
A(i, j)=\delta_{i j}-\cos (2 \pi(i-j) / M)
$$

where $\delta_{i j}$ is the Kronecker $\delta$ and $M$ is the number of outputs (Figure 1).

The A matrix is used to modify the response to the data based on the relation between the distances between the outputs. The outputs are thought of as located on a ring ('wraparound').

The network's operation is the standard negative feedback operation with lateral connections. It is illustrated in Figure 2 [equations (1)-(4)].

\subsection{Lateral connections}

Lateral connections have been derived from the rectified Gaussian distribution (Seung et al., 1998), which is a modified version of the standard Gaussian distribution in which the variables are constrained to be non-negative, enabling the use of non-convex energy functions. The standard Gaussian distribution may be defined by

$$
\begin{gathered}
p(y)=Z^{-1} e^{-\beta E(y)} \\
E(y)=\frac{1}{2} y^{T} A y-b^{T} y
\end{gathered}
$$

In which the quadratic energy function $E(y)$ is defined by the vector $b$ and the symmetric matrix $A$. The parameter $\beta=1 / T$ is an inverse temperature. Lowering the temperature concentrates the distribution at the minimum of the energy function. The factor $Z$ normalizes the integral of $p(y)$ to unity.

The cooperative distribution is chosen as its modes are closely spaced along a non-linear continuous manifold. The energy functions that can be used are those that block the directions in which the energy diverges towards negative infinity. Thus, the matrix has to fit the following property:

$$
y^{T} A y>0, \forall y: y_{i}>0, i=1 \ldots N
$$

In which, $N$ is the dimensionality of $y$. 


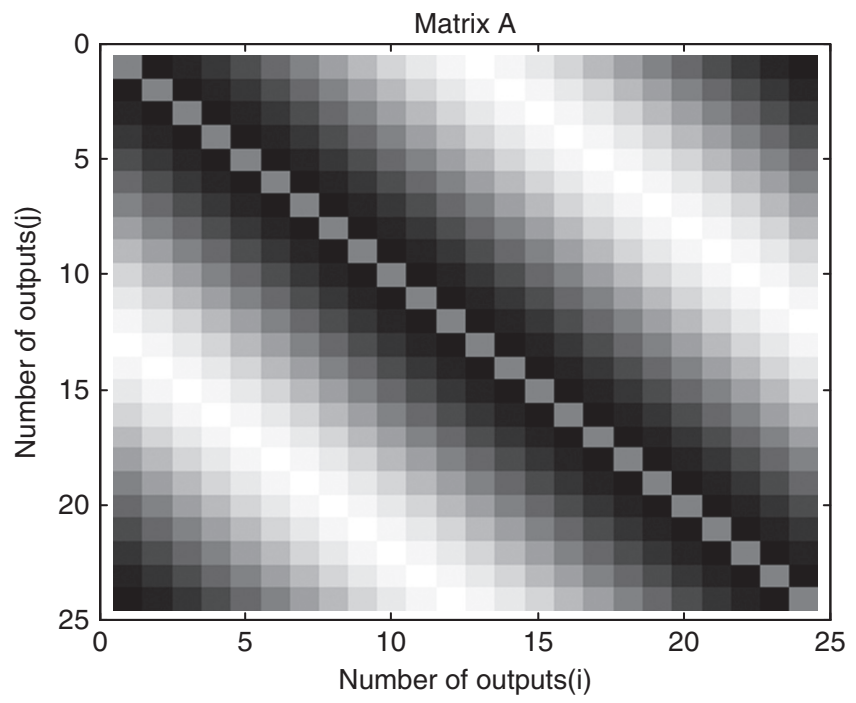

Figure 1: The A matrix for the rectified Gaussian network with 24 outputs. Black squares are negative, white are positive and the shading in each square is proportional to the weight size.

The cooperative distribution in the case of $N$ variables is defined by

$$
\begin{gathered}
A_{i j}=\delta_{i j}+\frac{1}{N}-\frac{4}{N} \cos \left(\frac{2 \pi}{N}(i-j)\right) \\
b_{i}=1
\end{gathered}
$$

In which $\delta_{i j}$ is the Kronecker $\delta$, and $i$ and $j$, the output neuron identifiers.

Matrix $A$ modifies the response to the data based on the relation between the distances between the outputs. The projected gradient method is used (Corchado et al., 2003), consisting of a gradient step followed by a rectification

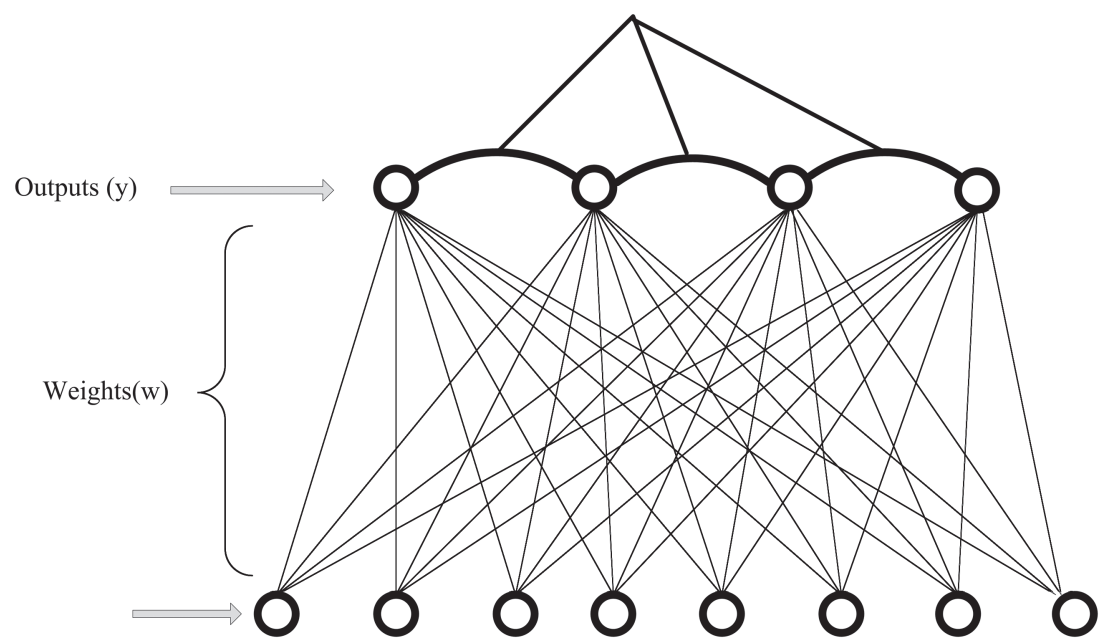

Figure 2: Lateral connections between neighbouring outputs. 
as specified in equation (2), in which the rectification []$^{+}$is necessary to ensure that the $y$-values remain within the positive quadrant. If the step size $(\tau)$ is chosen correctly, this algorithm will probably be shown to converge to a stationary point of the energy function (Bertsekas, 1999). In practice, this stationary point is generally a local minimum.

The distribution mode can be approached by gradient descent on the derivative of the energy function equation (11) with respect to $y$ :

$$
\Delta y \propto-\frac{\partial E}{\partial y}=-(A y-b)=b-A y
$$

The resulting model (CMLHL) can reveal the independent factors of a data set in a way that captures some type of global ordering in the data set and displays it with greater sparsity than other models.

Several versions of this model have successfully been applied to different data sets. Some of them are artificial, such as the well-known bars data set (Földiák, 1992; Corchado \& Fyfe, 2003) while others are real, such as data sets on banking, asteroids, algae (Corchado \& Fyfe, 2003) and knowledge management (Herrero et al., 2010).

\subsection{Fine tuning}

The CMLHL fine-tuning process is based on the effect of changing the $\tau$ parameter, which is the strength of the lateral connections between the output neurons. Experiments were conducted (Corchado \& Fyfe, 2003) using the bars data set (Földiák, 1992), which adds noise in a graduated manner across the outputs. These experiments showed that altering the strength of the lateral connection parameter modulated the ability of the neural network to 'gather' features together on the outputs. As predicted, a low $\tau$ value allows the neural model to code horizontal and vertical bars around a mode. An increase in the $\tau$ value means that the weak correlations between horizontal and vertical bars begin to have an impact on the learning. As the strength of the lateral connections becomes stronger, the bars are still learned around a mode but at the same time orientations start to separate. Subsequently, a separation emerges between the two different orientations, which is an interesting issue because all the data inputs to the network consist of both horizontal and vertical bars.

Increasing the $\tau$ value further will force the network to learn only one orientation of bars. However, if the lateral connections are too strong, then the coding of the bars may be squashed into an area of the output space that is too small for all of the bars to be coded individually. The reason why one orientation of bars is suppressed is due to the pixel overlap between different orientations of bars. If the lateral excitation between the output neurons is strong enough, a single output neuron may be able to switch its preference from a horizontal bar to a vertical one. That orientation identification was considered (Corchado \& Fyfe, 2003) to be a precursor of the creation of the concept of horizontal/vertical in animals inhabiting a mixed environment.

\section{Feature selection and extraction}

Feature selection and extraction (Guyon \& Elisseeff, 2003; Liu \& Yu, 2005) includes feature construction (Gavrilis et al., 2008), space dimensionality reduction (Liu et al., 2009b), sparse representations (Wright et al., 2009) and feature selection (Liu et al., 2009a). All these techniques are commonly used as pre-processing tools to machine learning tasks including pattern recognition. Although such problems have been tackled by researchers for many years, there has recently been a renewed interest in feature extraction. A large number of new applications with very large input spaces need space dimensionality reduction critically for the efficiency and efficacy of the predictors. Some of these applications include new and classical topics such as bioinformatics [DNA microarrays (Kim \& Cho, 2006; Gonzalez et al., 2009), remote sensing multi- and hyperspectral imagery (Malpica et al., 2008), pattern recognition [e.g., handwriting recognition (Su et al., 2009)], 
text processing (Valeriana-Garcia et al., 2008), Web mining (Chen et al., 2009), speech processing (Avci, 2007; Mostafa \& Billor, 2009), artificial vision (Raducanu et al., 2010), medical applications (Marinakis et al., 2009; Wolczowski \& Kurzynski, 2010), industrial applications (Avci et al., 2009)].

The approach taken to feature selection is based on space dimensionality reduction. It initially uses a projection method called CMLHL (Corchado \& Fyfe, 2003), which is characterized by its capability to enforce a sparser representation in each weight vector than other classical methods, such as PCA or MLHL.

The internal structures of complex clustering domains, such as high dimensional ones, may hinder their own internal structures or patterns. Such patterns may become visible if a change of basis of the space is made, however, an a priori decision as to which basis will reveal most patterns requires foreknowledge of the unknown patterns.

CMLHL is an EPP model aimed at solving the previous difficult problem of identifying structure in high-dimensional data by projecting the data onto a low-dimensional subspace in which its structure is searched for by eye. However, not all projections will reveal the data's structure equally well. Therefore, an index has been defined that measures how 'interesting' a given projection is; the data is represented in terms of projections that maximize that index. Interesting structure is usually defined with respect to the fact that most projections of high-dimensional data onto arbitrary lines through most multi-dimensional data give almost Gaussian distributions (Diaconis \& Freedman, 1984). Therefore, to identify 'interesting' features in data, directions should be looked for onto which the data projections are as far from the Gaussian as possible. CMLHL is based on the analysis of the kurtosis, which is based on the normalized fourth moment of the distribution and measures the heaviness of the tails of a distribution. A bimodal distribution will often have a negative kurtosis, meaning negative kurtosis can signal that a particular distribution shows evidence of clustering.

\section{System modelling using classical identification algorithms}

\subsection{Identification criterion}

The identification criterion consists in evaluating which of the group of candidate models is the best adapted and the one that best described the data set gathered for the experiment, i.e., given a certain model $M\left(\theta_{*}\right)$, its prediction error may be defined by equation (12). As stated in Ljung (1999), 'a good model is one that makes good predictions, and which produces small errors when the observed data is applied'. The estimated parametrical vector $\hat{\theta}_{N}$ is obtained in such a way that the prediction error $\varepsilon(t, \theta)$ is minimized for data set $Z^{t}$

$$
\varepsilon\left(t, \theta_{*}\right)=y(t)-\hat{y}\left(t \mid \theta_{*}\right)
$$

So, minimizing the error function $V_{N}\left(\theta, Z^{N}\right)$ generates the estimated parametrical vector $\hat{\theta}$. Typically, $V_{N}\left(\theta, Z^{N}\right)$ is calculated by the leastsquares criterion for the linear regression, i.e., by applying the quadratic norm $\ell(\varepsilon)=\frac{1}{2} \varepsilon^{2}$

$$
\begin{gathered}
V_{N}\left(\theta, Z^{N}\right)=\frac{1}{N} \sum_{t=1}^{N} \frac{1}{2}(y(t)-\hat{y}(t \mid \theta))^{2} \\
\hat{\theta}=\hat{\theta}_{N}\left(Z^{N}\right)=\underset{\theta \in D_{M}}{\arg \min } V_{N}\left(\theta, Z^{N}\right)
\end{gathered}
$$

One of the available methodologies of model structure is the black-box structures (Ljung, 1999), which has the advantage of only requiring very few explicit assumptions on the pattern to be identified, but that in turn makes it difficult to quantify the model that is obtained. The discrete linear models may be represented through the union of both deterministic and stochastic models. In equation (15), $u(t)$ is the input, $y(t)$ is the output, $G\left(q^{-1}\right)$ is the transfer function from $u(t)$ to $y(t), H\left(q^{-1}\right)$ is the transfer function from $e(t)$ to $y(t)$ and $q, q^{-1}$ are forward and backward shift operators. The term $e(t)$ (white noise signal) includes the modelling errors and is associated with a series of random variables of mean null value and variance $\lambda$

$$
y(t)=G\left(q^{-1}\right) u(t)+H\left(q^{-1}\right) e(t)
$$


The structure of a black-box model depends on how the noise influences the model (Ljung, 1999), that is, the term $H\left(q^{-1}\right)$. Thus, if this term is 1 , then the finite impulse response (FIR) (Fernandes et al., 2010) and output error (OE) (Taghavi \& Sadr, 2008; Gillberg \& Ljung, 2010) models are applicable; whereas if it is different from 0 a great range of models are applicable; the most common being: autoregressive with external input (ARX) (da Silva et al., 2009; Ismail et al., 2009), autoregressive moving average with external input (ARMAX) (Wang \& Cheng, 2009; Iqbal et al., 2010), box jenkins (BJ) (Meiler et al., 2008; Mustafaraj et al., 2010) and autoregressive moving average (ARMA) (Datong et al., 2009; Huang et al., 2009).

This structure may be represented in the form of a general model equations (16) and (18), where $B\left(q^{-1}\right)$ is a polynomial of degree $n_{b}$, which can incorporate pure delay $n_{k}$ in the inputs, and $A\left(q^{-1}\right), C\left(q^{-1}\right), D\left(q^{-1}\right)$ and $F\left(q^{-1}\right)$ are autoregressive polynomials of degree $n_{a}, n_{c}, n_{d}$ and $n_{f}$, respectively. In the same way, it is possible to use a predictor expression, for the one-step prediction ahead of the output $\hat{y}(t \mid \theta)$. The value of $n_{a}, n_{b}, n_{c}$, $n_{d}, n_{f}$ and $n_{k}$ are parameterized

$$
\begin{aligned}
A\left(q^{-1}\right) y(t) & =q^{-n_{k}} \frac{B\left(q^{-1}\right)}{F\left(q^{-1}\right)} u(t)+\frac{C\left(q^{-1}\right)}{D\left(q^{-1}\right)} e(t) \\
\hat{y}(t \mid \theta)= & \frac{D\left(q^{-1}\right) B\left(q^{-1}\right)}{C\left(q^{-1}\right) F\left(q^{-1}\right)} u(t) \\
& +\left[1-\frac{D\left(q^{-1}\right) A\left(q^{-1}\right)}{C\left(q^{-1}\right)}\right] y(t) \\
A\left(q^{-1}\right) & =\sum_{i=1}^{n_{a}} 1+a_{i}\left(q^{-i}\right), \\
B\left(q^{-1}\right) & =\sum_{i=1}^{n_{b}} b_{i}\left(q^{-i}\right), \\
C\left(q^{-1}\right) & =\sum_{i=1}^{n_{c}} 1+c_{i}\left(q^{-i}\right) \\
D\left(q^{-1}\right) & =\sum_{i=1}^{n_{d}} 1+d_{i}\left(q^{-i}\right) \\
F\left(q^{-1}\right) & =\sum_{i=1}^{n_{f}} 1+f_{i}\left(q^{-i}\right)
\end{aligned}
$$

\subsection{Modelling the laser-milling optimal conditions}

This study tries to find the best model for estimating the optimal conditions in a high precision laser-milling process. An identification procedure should be used so the experimentation can be carried out for different cases. As stated in Haber and Keviczky (1999a, 1999b), Ljung (1999), Nørgaard et al. (2000) and Nelles (2001), the identification procedure includes establishing the identification techniques, the selection of the model structure, the estimation of the suitable polynomials degree, choosing the identification criterion and the optimization techniques to generate the final model.

Also, the identification procedure includes the training and the validation stages, which ensures that the selected model meets the necessary conditions for estimation and prediction. In order to validate the model, three tests were performed: the residual analysis $\varepsilon(t, \hat{\theta}(t))$ by means of a correlation test between inputs, the final prediction error (FPE) estimate as explained by Akaike (1969) and lastly the graphical comparison between desired outputs and the outcome of the models through simulation 1 (or k) steps before.

\section{An industrial case study: choosing the optimal operating conditions}

In this study, a procedure to determine the optimal operating conditions for a laser-milling process is described. The procedure includes three steps, as shown in Figure 3. After data set gathering, in the first step, an analysis of the data set is performed to identify if it is informative enough. If the gathered data set is not valid, then it should be discarded and a new data set should be considered. The second step is based on feature selection to identify the most relevant variables; its outcome is the dimensional reduced data set. Finally, the third step involves searching for the model that best suits fits the operating conditions; its outcome is the model to be used, finding the best operating conditions in each case. 


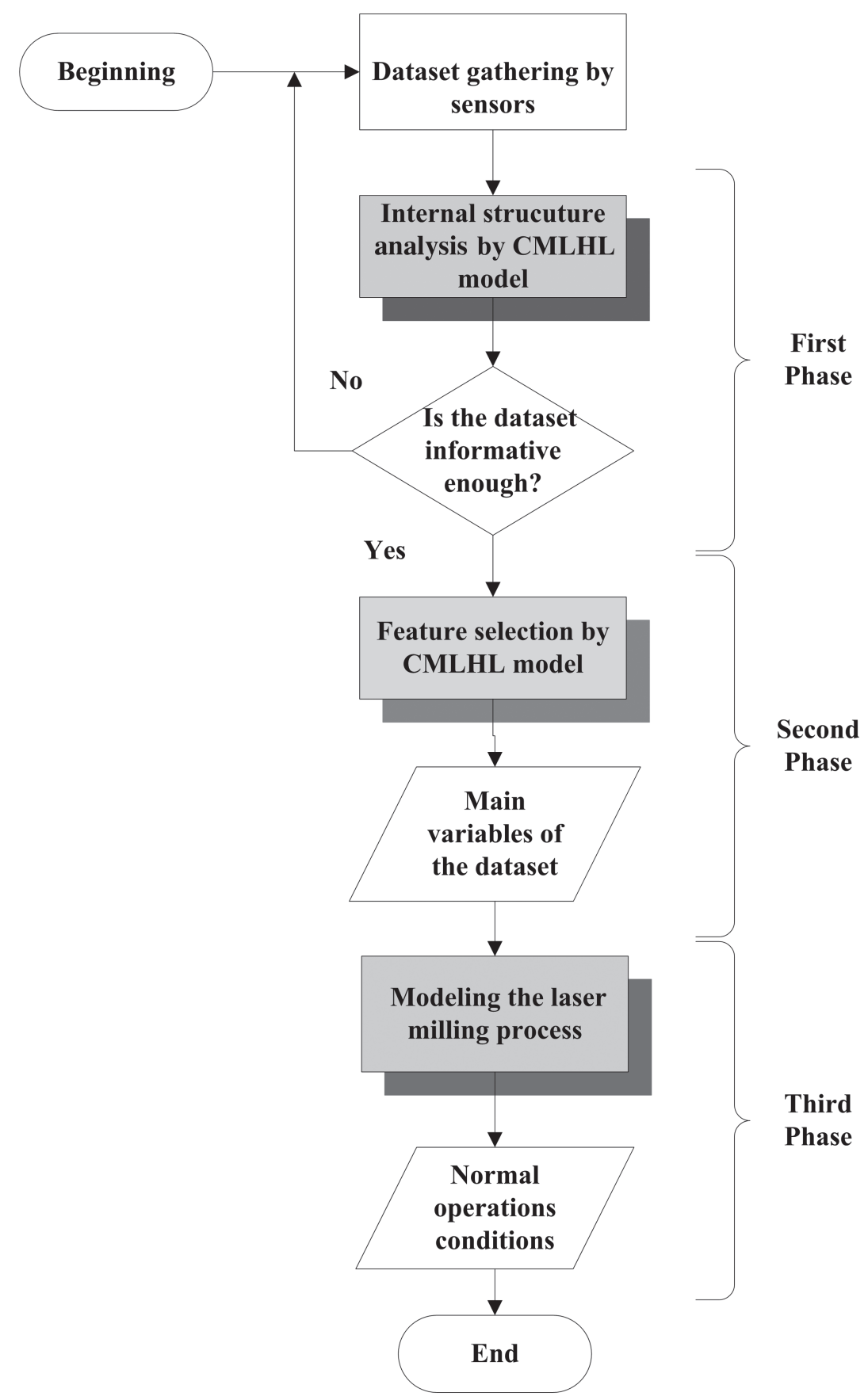

Figure 3: The flow chart of the proposed procedure.

The procedure is validated against three, common, real-world laser-milling problems in the industry. The first one is copper, a material used in the manufacture of electrodes for EDM. The second one is aluminium, a material commonly used for highly complex moulds for 
medical applications. The third one is hardened steel, which is often used in the automotive industry, where laser milling allows the deep marking of serial numbers or barcodes for quality control. These three materials cover a broad range of industrial applications of laser milling and micro-manufacturing.

Modelling the laser-milling process involves several steps. After a data set is collected through the use of sensors, an internal structure analysis is carried out. The most significant variables then have to be identified. Finally, the model must be generated considering the most important variables and the relationships found. These steps are detailed below.

\subsection{Data set generation}

To describe the industrial problem, a test piece has been designed. The test piece is an inverted truncated pyramid profile that is to be laser milled on a flat metallic piece of the three selected materials. The truncated pyramid angles are theoretically of $135^{\circ}$ and the depth (or height) of the truncated pyramid is $1 \mathrm{~mm}$, but as the laser parameters are not known for these materials, both parameters will show an error on the real machined pieces called angle error and depth error, referred in this paper as $y_{1}$ and $y_{2}$, respectively. The prediction of the geometrical error through these two variables is enough to assure the geometrical quality of the micro-tools that will be machined by laser manufacturing.

The test piece was laser milled using a laser with a pulse length of $10 \mu \mathrm{s}$. Some parameters of the laser process can be controlled: the laser power $\left(u_{1}\right)$, the laser-milling speed $\left(u_{2}\right)$, the laser spot diameter, the distance from the laser focus to the piece (positioning along the $Z$-axis adjustment), the machining strategy and the laser pulse frequency $\left(u_{3}\right)$. It is important to note that all these parameters are standard data provided by the laser-milling centre, so the industrial implementation of the model will be immediate. For the data analysis, three other variables related to the milled material were also considered: thermal conductivity, reflectivity and density.
The experiment design included variation of all the parameters mentioned above, with the exception of laser spot diameter and machining strategy, which were constant for all tests. Almost 100 different experiments were carried out, which meant a large increase in the cost of the study. After the laser milling of the test piece previously described, the actual inverted pyramid depth and the wall angle were measured by means of proper optical measurements. These measurements were compared with theoretical values $\left(135^{\circ}\right.$ and $1 \mathrm{~mm}$, respectively) and the difference between theoretical and experimental values represents the geometrical errors of the machined piece: angle error $\left(y_{1}\right)$ and depth error $\left(y_{2}\right)$. Both geometrical errors $-y_{1}$ and $y_{2}-$ are considered as output parameters of each experiment.

\subsection{The first two steps: extracting the relevant internal structures and main variables (feature selection)}

\subsubsection{Analysing the internal structure of a data} set As detailed in Section 2, PCA and CMLHL are two methods for identifying the internal structure of the data; both were applied to this industrial problem. Both methods have been applied to the three different case studies to know if the data sets are informative enough and also identified the most interesting underlying variables.

The following figures show the results of applying PCA [Figures 4(a), 5(a) and 6(a)] and CMLHL [Figures 4(b), 5(b) and 6(b)] in three different cases study. The vertical and horizontal axes forming these projections are combinations of the variables contained in the original data sets.

By using CMLHL [Figure 4(b)], it has been obtained a more sparse representation than with PCA [Figure 4(a)]. It can be easily seen how each group is formed by another three sub-groups and that the samples are clearly grouped and separated. CMLHL has identified three different groups or clusters [Figure 4(b)] order by speed. After studying each cluster it is noted a second classification, which is based on the 

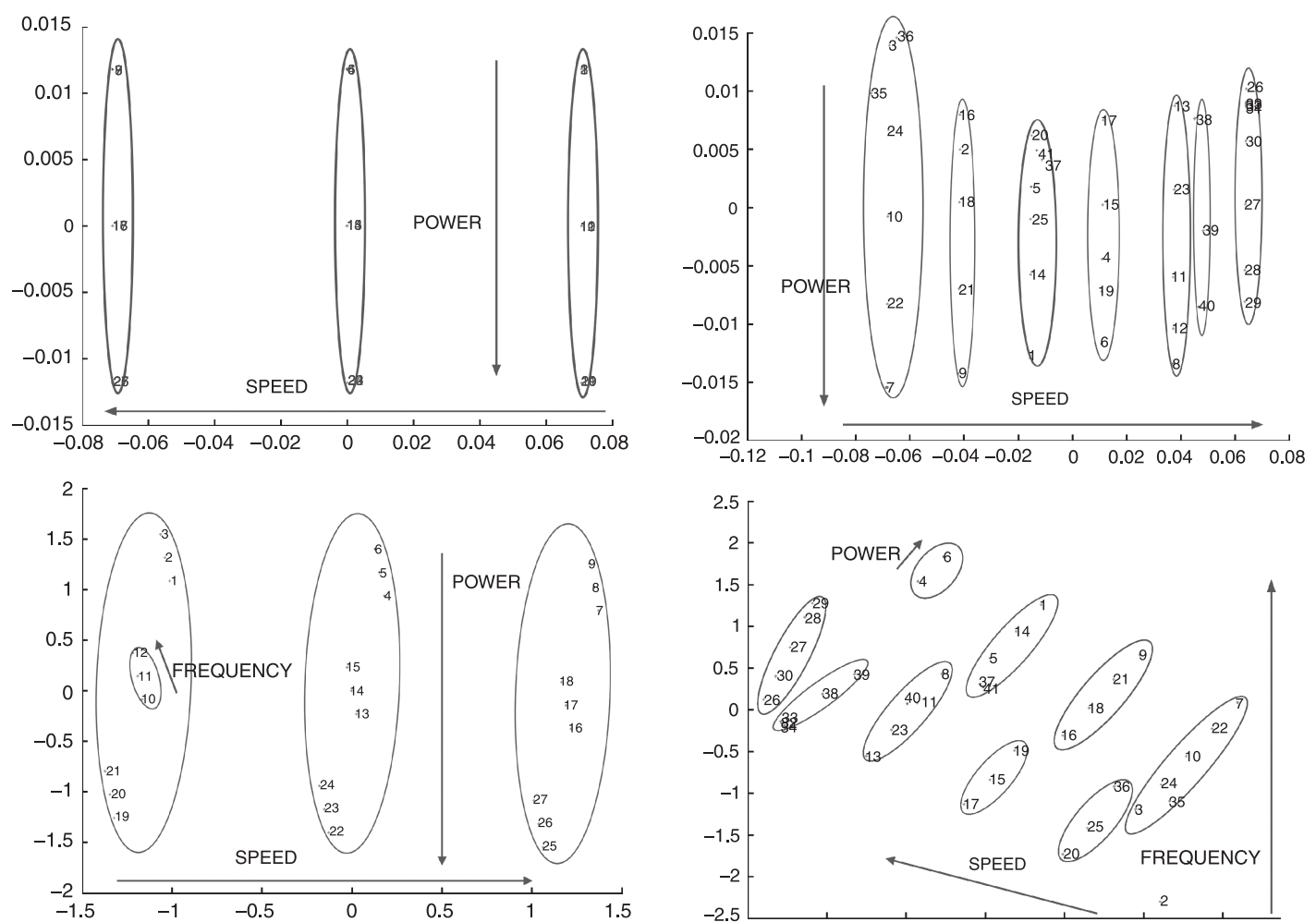

Figure 4: $\quad P C A$ projections (a) and CMLHL projections (b) for a steel piece.

speed and frequency as it is shown in the right side of Figure 4(b).

CMLHL [Figure 5(b)] has identified several clusters ordered by speed for aluminium components. It is worthy to note that, again, CMLHL is providing a more sparse visualization than PCA [Figure 5(a)] and that this method has identified several clusters ordered by speed and frequency, and inside each cluster ordered by power.

As in the previous cases, it can be seen how CMLHL [Figure 6(b)] has identified different cluster ordered by speed. For this material, five clusters have been identified and inside each clusters it is possible to notice another classification by frequency and power [Figure 6(b)]. Yet again, the use of PCA [Figure 6(a)] is providing a five cluster projection but in less sparse and informative way than CMLHL [Figure 6(b)].

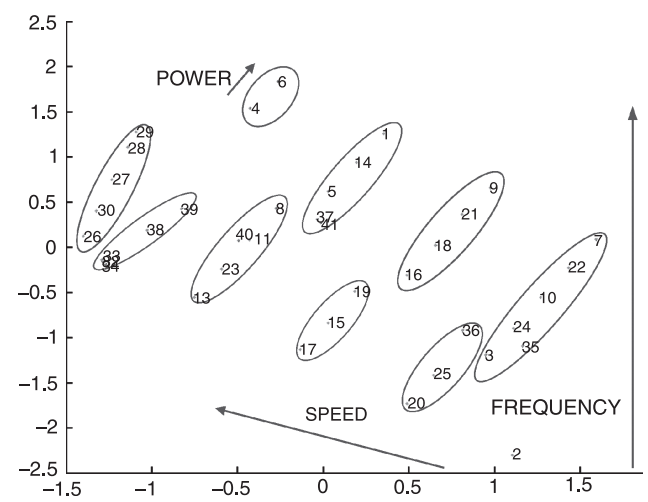

Figure 5: $P C A$ projections (a) and CMLHL projections (b) for an aluminium component.

As it can be seen in the previous figures (Figures 4-6), both methods have identified a clear internal structure in the case of the three different materials as several well-defined clusters have been identified. It can be affirmed that CMLHL provides, in general, a sparser representation than PCA due to the combined use of MLHL-based method and the application of lateral connections. As it is clear that there are several well-defined groups, the three data sets describing each material are informative enough, and it is possible to move to the second step of this model.

5.2.2. Feature selection by CMLHL By analysing the results obtained by CMLHL [Figures 4(b), 5(b) and 6(b)] of the three materials in the second step, it can be seen that, of the original 

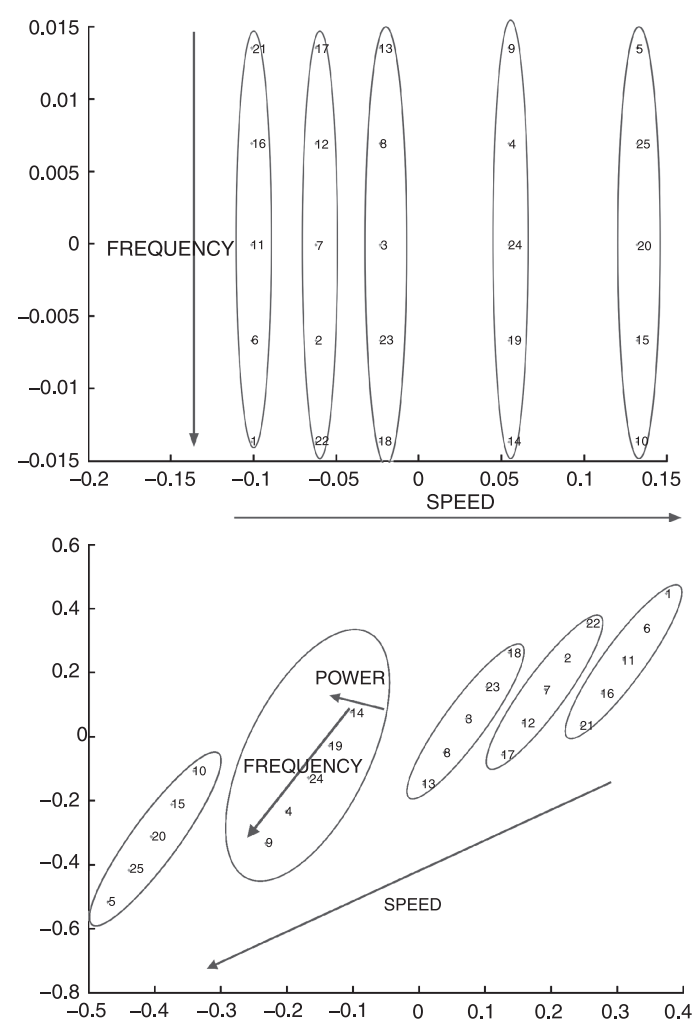

Figure 6: $P C A$ projections (a) and CMLHL projections (b) for a copper piece.

data sets, the most significant variables to be processed in the third step are: power, speed and frequency. This leads to the application of the third phase or step of this process, which accurately and efficiently optimizes the model of the laser milling by applying several classical modelling systems.

Thus, for these three materials, the date sets describing each element are informative enough (first step). The main variables to be analysed (second step) in the third and final step of the presented model are the power, the speed and the frequency.

\subsection{The third step: applying system identification for modelling the laser milling optimal conditions}

The different model learning methods used were implemented in Matlab $^{\odot}$ making use of its toolboxes - function libraries for Matlab: the system identification toolbox and the control system toolbox. The experiment followed the identification procedure detailed in Section 4.2: the model structures were analysed to obtain the models that best suite the data set. The Akaike information criterion (AIC) was used to obtain the best degree of the model and its delay for each model structure. A total of 36 techniques were carried out to obtain the models, including:

- The frequency response analysis based on the spectrum analysis and the Fourier fast transform (FFT) were used to determine the data dynamics.

- The FIR method correlation analysis was used to determine the steady state conditions.

- The black-box models synthesis: up to 31 different combinations of model structure and optimization technique were considered, such as the least-squares method, the QR factorization of ARX models and the recursive normalized gradient algorithm of RARMAX models (Söderström \& Stoica, 1989; Ljung, 1999).

- Three different residual analysis based on cross correlation were carried out: the residual analysis between the residual $\hat{R}_{\varepsilon}^{N}(\tau)$, between the residual and the input $\hat{R}_{\varepsilon u}^{N}(\tau)$, and the non-linear residual correlation $\hat{R}_{\varepsilon^{2} u^{2}}^{N}(\tau)$.

To validate the obtained models, several different indexes have been used. The indexes are recognized and widely used measures in system identification (Söderström \& Stoica, 1989; Ljung, 1999; Nørgaard et al., 2000).

- The percentage representation of the estimated model. This index is calculated as the normalized mean error for the one-step prediction (FIT1), for the ten-step prediction (FIT10) and with the $\infty$-step prediction (FIT). The FIT is known as simulation in classical system identification.

- The graphical representation of the FIT1 $\hat{y}_{1}(t \mid m),-$ the FIT10 $-\hat{y}_{10}(t \mid m)-$ and the FIT $-\hat{y}_{\infty}(t \mid m)$. 
- The loss function or error function $(V)$ : the numeric value of the mean-squared error (MSE) that is computed with the estimation data set.

- The generalization error value: the numeric value of the normalized sum of squared errors (NSSE) that is computed with the validation data set.

- The FPE is calculated as the average generalization error value computed with the estimation data set.

The results of modelling each of the three industrial processes are shown from Figures 7 9 for copper, aluminium and steel, respectively. The Figures 7-9 show the graphical representations for the best models found in each case. In all of them, the $X$-axis represents the number of samples used in the validation of the model, while the $Y$-axis represents the normalized output variable range - the normalization removes just the mean value -, with the output variable being the angle error - in degrees - or the depth error - in millimeters - of the test piece. In all the figures, the real operation condition is plotted as a solid line, and the estimated output of the model is plotted as a dotted line. The training and the validation data sets include 78 and 20 samples, respectively.

For milling copper components, the best models found for both the angle error (see
Table 1) and the depth error (see Table 2) are the ARX and the OE models, which are found to be totally equivalent according also to the results in Figure 7. These models not only present the lower loss function and generalization error values, but also the higher system representation indexes (FIT and FIT1). Finally, the polynomials parameters for the OE and the ARX models are presented in Tables 3 and 4, respectively.

The same reasoning is followed for the aluminium and the hardened steel components. The best models found indexes values are presented in Tables 5 and 6 for the aluminium components and in Tables 9 and 10 for hardened steel, for the angle and depth errors, respectively. In the case of aluminium components, the best models found are the $\mathrm{OE}$ and $\mathrm{BJ}$ models shown in Tables 7 and 8 .

From the graph (Figure 8), it can be concluded that the BJ model is the best model for simulating and predicting the behaviour of the laser milled with an aluminium test piece for both outputs: the angle error and the depth error, as they meet the indicators and are capable of modelling more than $99 \%$ of the true measurements.

For hardened steel components, the best models are presented in Tables 9 and 10 for the angle error and the depth error, respectively; while Figure 9 shows the one-step prediction
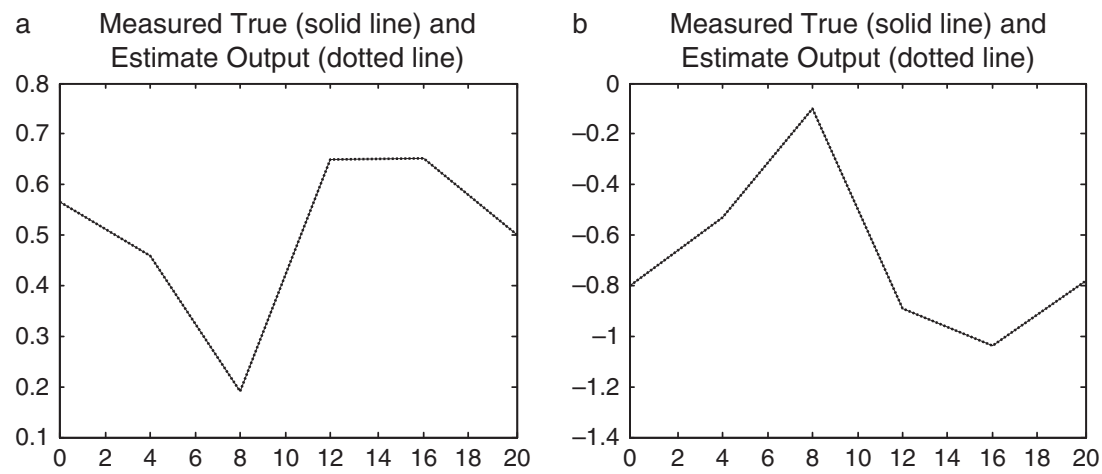

Figure 7: Milling of copper components: (a) OE and ARX models for the angle error; ( $b$ ) $O E$ and $A R X$ models for the depth error. The real measurement (solid line), the simulated output and the onestep prediction (dotted line) for $O E$ and $A R X$ models are shown. 

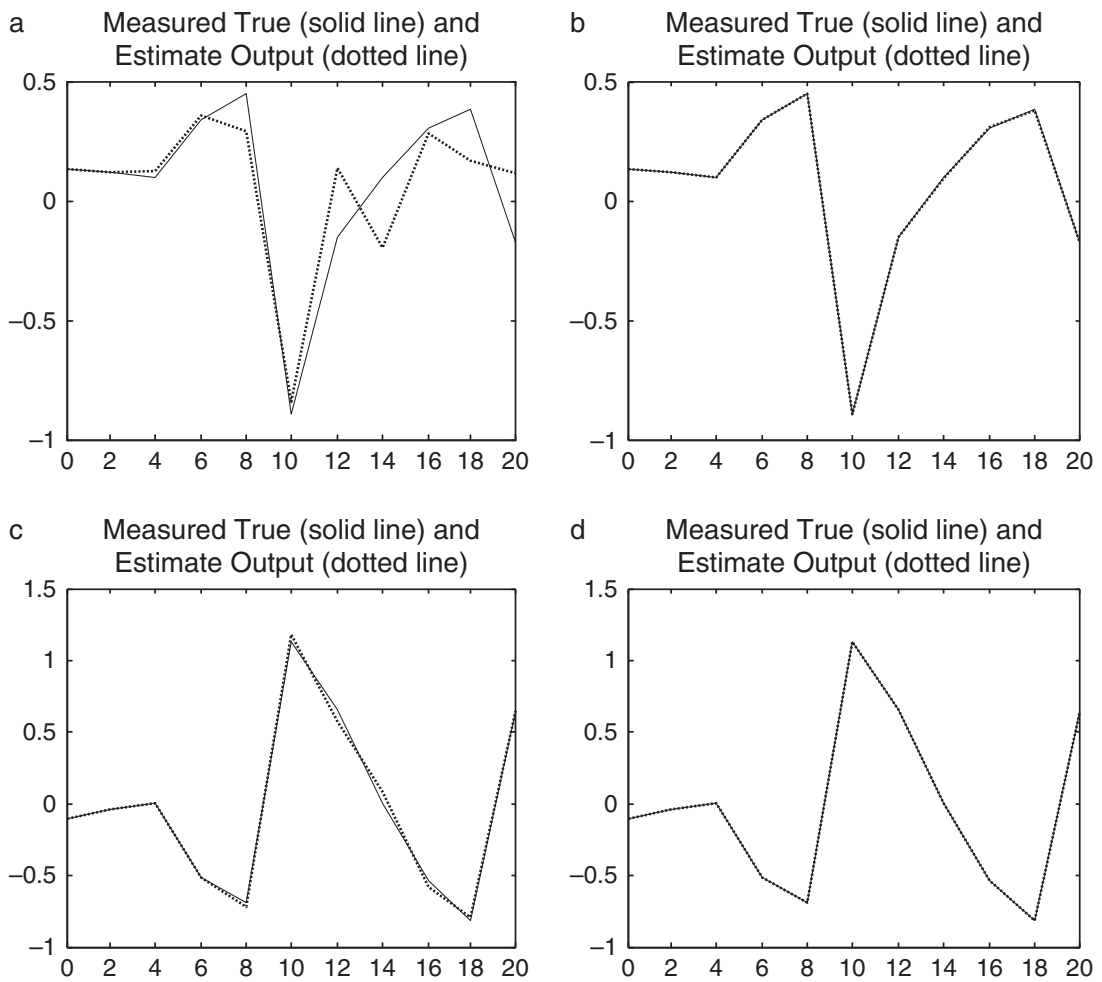

Figure 8: Milling of aluminium components: (a) OE model for the angle error; (b) BJ model for the angle error; (c) OE model for the depth error; (d) BJ model for the depth error. Representation of the real measurement (solid line), the simulated output and the one-step prediction (dotted line) for $O E$ and BJ models.

and the simulated output for the $\mathrm{OE}$ and the $\mathrm{BJ}$ models.

From the graph [Figures 9(a) and 9(b)], it can be concluded that the $\mathrm{BJ}$ model is the best model for simulating and predicting the behaviour of the laser-milled test piece of steal for angle error better than the OE model. Also, the BJ and OE models [Figure 9(c) and 9(d)] are capable of simulating and predicting the behaviour of the laser-milled piece of steal for depth error in the same manner (see also Table 10). All these models are capable of modelling more than $99 \%$ of the true measurements. The comparison of the best models found is shown in Tables 9 and 10 by model function and type. The chosen BJ and OE models are detailed in Tables 11 and 12 .

The obtained models can be used not only to predict the angle error and the depth error of the test piece, but also to determine the optimal conditions to minimize the error: considering that it is a polynomial model, if all but one input variable are fixed, the remaining variables could be calculated and fixed to minimize the angle error and the depth error of the test piece on the flat metallic piece of copper, aluminium and steel. So, in Figure 10, a graph of the errors in the flat metallic piece of aluminium is shown related to the others three input components: power, speed and frequency.

Figure 10 shows the output response of the two different errors: the angle error [Figure 10(a)] and depth error [Figure 10(b)] for different input variable ranges. The angle error and the depth can be zero for different values of power and speed for a constant value of frequency; i.e., it is possible to achieve an angle error close to 0 for a laser power of $60 \%$ and a 

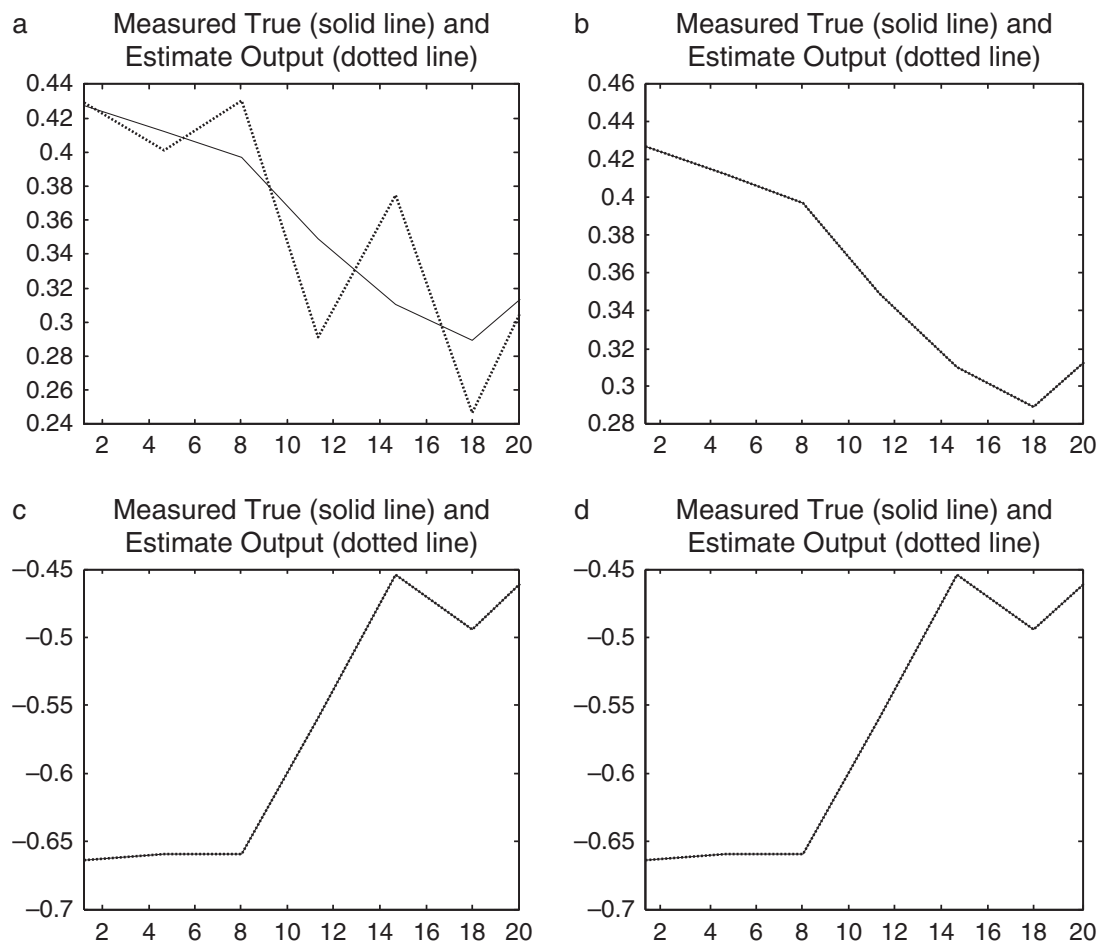

Figure 9: Milling hardened steel components: (a) OE model for the angle error; (b) BJ model for the angle error; (c) OE model for the depth error; (d) BJ model for the depth error. Representation of the real measurement (solid line), the simulated output and the one-step prediction (dashed line) for OE and BJ models.

milling speed of $460 \mathrm{~mm} / \mathrm{s}$. The $X$-axis shows the variable range of power $u_{1}(t)$, from 50 to 100 , as a percentage of the maximum power performed by the laser $(\%)$ and the $Y$-axis represents the variable range of speed $u_{2}(t)$, from 225 to 525 , in $\mathrm{mm} / \mathrm{s}$. The variable frequency $u_{3}(t)$ is fixed at $85 \mathrm{kHz}$. The errors of the test piece are shown on the bars, which are distributed from $-0.4^{\circ}$ to $0.1^{\circ}$ and from -0.05 to $0.25 \mathrm{~mm}$ for the angle error $y_{1}(t)$ and the depth error $y_{2}(t)$, respectively.

\section{Conclusions and future work}

This interdisciplinary research has presented a detailed study for designing a three-step soft computing procedure to identify the most appropriate modelling system to solve a real-life high precision industrial problem: the laser milling of metal components. The procedure has been validated with three different materials: aluminium, copper and hardened steel. It is worth mentioning that with classical and soft computing techniques, two interesting variables such as the angle error and the depth error have been successfully modelled.

The purpose of this solution is to assist endusers in choosing the correct operating conditions of the tools, in this case, a laser mill. The process data analysis included in this procedure enables the users to apply this solution in different scenarios (i.e., in dental milling, heater system, incremental deformation, diagnostic system, failure detection systems, water consumption prediction, etc.). The expected results include a reduced number of input variables due to feature selection or extraction, using the most 


\begin{tabular}{|c|c|}
\hline Model & Performance indexes \\
\hline $\begin{array}{l}\text { Black-box OE model with } n_{b 1}=1, n_{b 2}=4, n_{b 3}=1, n_{f}=1, n_{k 1}=1, n_{k 2}=2 \text {, } \\
n_{k 3}=1 \text {. The model is estimated using the prediction error method; the } \\
\text { degree of the model selection is carried out from the best AIC criterion } \\
\text { (the structure that minimizes AIC) }\end{array}$ & $\begin{array}{l}\text { FIT: } 100 \% \text {, FIT1: } 100 \% \\
\text { FIT10: } 100 \% \text {, V: } 0.066 \\
\text { FPE: } 1.1302 \text {, NSSE: } 7.46 \mathrm{e}-31 \\
\text { Variance of } e(t): 0.598\end{array}$ \\
\hline $\begin{array}{l}\text { Black-box OE model with } n_{b 1}=1, n_{b 2}=4, n_{b 3}=1, n_{f}=1, n_{k 1}=1, n_{k 2}=3 \text {, } \\
n_{k 3}=1 \text {. The model is estimated using the prediction error method; the } \\
\text { degree of the model selection is carried out with the best AIC criterion } \\
\text { (the structure that minimizes AIC) }\end{array}$ & $\begin{array}{l}\text { FIT: } 100 \% \text {, FIT } 1: 100 \% \\
\text { FIT10: } 100 \% \text {, V: } 0.011 \\
\text { FPE: } 0.413 \text {, NSSE: } 3.76 \mathrm{e}-30 \\
\text { Variance of } e(t): 0.212\end{array}$ \\
\hline $\begin{array}{l}\text { Black-box ARX model with } n_{a}=1, n_{b 1}=1, n_{b 2}=4, n_{b 3}=1, n_{k 1}=1 \text {, } \\
n_{k 2}=2, n_{k 3}=1 \text {. The model is estimated using the least squares method, } \\
\text { QR factorization; the degree of the model selection is carried out with the } \\
\text { best AIC criterion (the structure that minimizes AIC) }\end{array}$ & $\begin{array}{l}\text { FIT: } 18.34 \% \text {, FIT1: } 11.13 \% \\
\text { FIT10: } 11.13 \%, \text { V: } 0.066 \\
\text { FPE: } 0.1514 \text {, NSSE: } 0.019\end{array}$ \\
\hline $\begin{array}{l}\text { Black-box ARX model with } n_{a}=1, n_{b 1}=1, n_{b 2}=4, n_{b 3}=1, n_{k 1}=1 \text {, } \\
n_{k 2}=3, n_{k 3}=1 \text {. The model is estimated using the least squares method, } \\
\text { QR factorization; the degree of the model selection is carried out with the } \\
\text { best AIC criterion (the structure that minimizes AIC) }\end{array}$ & $\begin{array}{l}\text { FIT: } 100 \% \text {, FIT } 1: 100 \% \\
\text { FIT10: } 100 \% \text {, V: } 0.061 \\
\text { FPE: } 0.139 \text {, NSSE: } 1.68 \mathrm{e}-28 \\
\text { Variance of } e(t): 0.22\end{array}$ \\
\hline
\end{tabular}

Indicator values for several proposed models of the angle error.

Table 2: $\quad$ Milling of copper components

\begin{tabular}{|c|c|}
\hline Model & Performance indexes \\
\hline $\begin{array}{l}\text { Black-box OE model with } n_{b 1}=1, n_{b 2}=4, n_{b 3}=1, n_{f}=1, n_{k 1}=1, n_{k 2}=2 \text {, } \\
n_{k 3}=1 \text {. The model is estimated using the prediction error method; the } \\
\text { degree of the model selection is carried out from the best AIC criterion } \\
\text { (the structure that minimizes AIC) }\end{array}$ & $\begin{array}{l}\text { FIT: } 100 \%, \text { FIT } 1: 100 \% \\
\text { FIT10: } 100 \%, \text { V: } 0.083 \\
\text { FPE: } 1.42 \text {, NSSE: } 1.21 \mathrm{e}-29 \\
\text { Variance of e(t): } 0.755\end{array}$ \\
\hline $\begin{array}{l}\text { Black-box OE model with } n_{b 1}=1, n_{b 2}=4, n_{b 3}=1, n_{f}=1, n_{k 1}=1, n_{k 2}=3 \text {, } \\
n_{k 3}=1 \text {. The model is estimated using the prediction error method; the } \\
\text { degree of the model selection is carried out with the best AIC criterion } \\
\text { (the structure that minimizes AIC) }\end{array}$ & $\begin{array}{l}\text { FIT: } 100 \%, \text { FIT1: } 100 \% \\
\text { FIT10: } 100 \% \text {, V: } 0.029 \\
\text { FPE: } 1.047 \text {, NSSE: } 2.63 \mathrm{e}-29 \\
\text { Variance of e(t): } 0.538\end{array}$ \\
\hline $\begin{array}{l}\text { Black-box ARX model with } n_{a}=1, n_{b 1}=1, n_{b 2}=4, n_{b 3}=1, n_{k 1}=1 \text {, } \\
n_{k 2}=2, n_{k 3}=1 \text {. The model is estimated using the least squares method, } \\
\text { QR factorization; the degree of the model selection is carried out with the } \\
\text { best AIC criterion (the structure that minimizes AIC) }\end{array}$ & $\begin{array}{l}\text { FIT: } 43.58 \% \text {, FIT1: } 42.33 \% \\
\text { FIT10: } 42.33 \% \text {, V: } 0.101 \\
\text { FPE: } 0.2315 \text {, NSSE: } 0.0308\end{array}$ \\
\hline $\begin{array}{l}\text { Black-box ARX model with } n_{a}=1, n_{b 1}=1, n_{b 2}=4, n_{b 3}=1, n_{k 1}=1 \text {, } \\
n_{k 2}=3, n_{k 3}=1 \text {. The model is estimated using the least squares method, } \\
\text { QR factorization; the degree of the model selection is carried out with the } \\
\text { best AIC criterion (the structure that minimizes AIC) }\end{array}$ & $\begin{array}{l}\text { FIT: } 100 \% \text {, FIT1: } 100 \% \\
\text { FIT10: } 100 \%, \text { V: } 0.087 \\
\text { FPE: } 0.198 \text {, NSSE: } 3.60 \mathrm{e}-30 \\
\text { Variance of e(t): } 0.313\end{array}$ \\
\hline
\end{tabular}

Indicator values for several proposed models of the depth error.

informative features, so the complexity of the model is relaxed. Previous contributions in the literature are usually based in the prior process knowledge - physical system information -, which is not always feasible.

In the case of laser milling, the amount of material removed is not only unknown as it depends on the laser pulse characteristics, but also difficult to estimate. Different approaches for estimating such variable - this is the amount of material removed - have been published, though they are based in a priori specific knowledge of the system. Up to our knowledge, these solutions were not valid as 
Parameters and polynomials

\begin{tabular}{ll}
\hline$B 1(q)=0.03695 q^{-1}$ & $F 1(q)=1+0.6718 q^{-1}$ \\
$B 2(q)=-0.0001911 q^{-3}+0.000186 q^{-4}$ & $F 2(q)=1+0.5765 q^{-1}$ \\
$-0.0002806 q^{-5}+0.001646 q^{-6}$ & $F 3(q)=1+0.9986 q^{-1}$ \\
$B 3(q)=-0.01592 q^{-1}$ & $e(t)$ is white noise signal \\
& with variance 0.21 \\
\hline
\end{tabular}

Function and parameters that represent the behaviour of the laser-milled piece for the angle error. The degree of the OE model polynomials are $n_{b 1}=1, n_{b 2}=4, n_{b 3}=1, n_{f}=1, n_{k 1}=1, n_{k 2}=3, n_{k 3}=1$. [ [ $\left.\begin{array}{llllllll}4 & 1 & 1 & 1 & 3 & 1\end{array}\right]$.

Table 4: $\quad$ Milling of copper components

\begin{tabular}{ll}
\hline & Parameters and polynomials \\
\hline$A 1(q)=1+0.5261 q^{-1}$ & $B 1(q)=-0.04465 q^{-1}$ \\
$B 2(q)=0.0006061 q^{-3}$ & $B 3(q)=0.01051 q^{-1}$ \\
$+0.0002783 q^{-4}+0.0001222 q^{-5}$ & $e(t)$ is white noise signal with variance 0.31 \\
$-0.001414 q^{-6}$ &
\end{tabular}

Function and parameters that represent the behaviour of the laser-milled piece for the depth. The degree of the ARX model polynomials are $n_{a}=1, n_{b 1}=1, n_{b 2}=4, n_{b 3}=1, n_{k 1}=1, n_{k 2}=3, n_{k 3}=1$. [ [ $\left.\begin{array}{lllllll}1 & 1 & 4 & 1 & 1 & 3 & 1\end{array}\right]$.

Table 5: Milling of aluminium components. Indicator values for several proposed models of the angle error

\begin{tabular}{|c|c|}
\hline Model & Performance indexes \\
\hline $\begin{array}{l}\text { Black-box OE model with } n_{b 1}=2, n_{b 2}=2, n_{b 3}=1, n_{f}=2, n_{k 1}=2, n_{k 2}=2 \text {, } \\
n_{k 3}=1 \text {. The model is estimated using the prediction error method; the } \\
\text { degree of the model selection is carried out from the best AIC criterion } \\
\text { (the structure that minimizes AIC) }\end{array}$ & $\begin{array}{l}\text { FIT: } 30.73 \%, \text { FIT } 1: 30.73 \% \\
\text { FIT10: } 30.73 \%, \text { V: } 0.117 \\
\text { FPE: } 0.471, \text { NSSE: } 0.0617\end{array}$ \\
\hline $\begin{array}{l}\text { Black-box OE model } n_{b 1}=3, n_{b 2}=1, n_{b 3}=1, n_{f}=2, n_{k 1}=2, n_{k 2}=1 \text {, } \\
n_{k 3}=1 \text {. The model is estimated using the prediction error method; the } \\
\text { degree of the model selection is carried out with the best AIC criterion } \\
\text { (the structure that minimizes AIC) }\end{array}$ & $\begin{array}{l}\text { FIT: } 51.76 \%, \text { FIT1: } 51.76 \% \\
\text { FIT10: } 51.76 \% \text {, V: } 0.1932 \\
\text { FPE: } 0.80, \text { NSSE: } 0.0299\end{array}$ \\
\hline $\begin{array}{l}\text { Black-box BJ model with } n_{b 1}=2, n_{b 2}=2, n_{b 3}=1, n_{c}=3, n_{d}=2, n_{f}=2, \\
n_{k 1}=2, n_{k 2}=2, n_{k 3}=1 \text {. The model is estimated using the prediction error } \\
\text { method; the degree of the model selection is carried out with the best AIC } \\
\text { criterion (the structure that minimizes AIC) }\end{array}$ & $\begin{array}{l}\text { FIT: } 44.44 \%, \text { FIT1: } 64.41 \% \\
\text { FIT10: } 36.81 \%, \text { V: } 0.053 \\
\text { FPE: } 0.588, \text { NSSE: } 0.016\end{array}$ \\
\hline $\begin{array}{l}\text { Black-box BJ model with } n_{b 1}=3, n_{b 2}=1, n_{b 3}=1, n_{c}=3, n_{d}=2, n_{f}=2, \\
n_{k 1}=2, n_{k 2}=1, n_{k 3}=1 \text {. The model is estimated using the prediction error } \\
\text { method; the degree of the model selection is carried out with the best AIC } \\
\text { criterion (the structure that minimizes AIC) }\end{array}$ & $\begin{array}{l}\text { FIT: } 99.53 \%, \text { FIT1: } 99.41 \% \\
\text { FIT10: } 99.53 \%, \text { V: } 0.104 \\
\text { FPE: } 1,46, \text { NSSE: } 4.49 \mathrm{e}-6\end{array}$ \\
\hline
\end{tabular}

they used unobservable variables from the process.

Nevertheless, this novel proposal allows human operators to provide values for a small number of input variables out of the whole input set and to obtain, by using in this case of study the obtained model, the angle and the depth values of the tool or piece, which are the final operating parameters and the most difficult ones to estimate. Thus, an important decrease in 
Table 6: Milling of aluminium components. Indicator values for several proposed models of the depth error

\begin{tabular}{ll}
\hline Model & Performance indexes \\
\hline $\begin{array}{l}\text { Black-box OE model with } n_{b 1}=2, n_{b 2}=2, n_{b 3}=1, n_{f}=2, n_{k 1}=2, n_{k 2}=2, \\
n_{k 3}=1 \text {. The model is estimated using the prediction error method; the } \\
\text { degree of the model selection is carried out from the best AIC criterion } \\
\text { (the structure that minimizes AIC) }\end{array}$ & $\begin{array}{l}\text { FIT: } 61.09 \%, \text { FIT1: } 61.09 \% \\
\text { FIT10: } 61.09 \%, \text { V: } 0.296\end{array}$ \\
$\begin{array}{l}\text { Black-box OE model with } n_{b 1}=1, n_{b 2}=3, n_{b 3}=1, n_{f}=2, n_{k 1}=1, n_{k 2}=3, \\
n_{k 3}=1 . \text { The model is estimated using the prediction error method; the }\end{array}$ & FIT: $92.98 \%$, FIT1: $92.98 \%$ \\
degree of the model selection is carried out with the best AIC criterion & FIT10: $92.98 \%$, V: 0.174 \\
(the structure that minimizes AIC) & FPE: 0.874, NSSE: 0.0017 \\
$\begin{array}{l}\text { Black-box BJ model with } n_{b 1}=2, n_{b 2}=2, n_{b 3}=1, n_{c}=3, n_{d}=2, n_{f}=2, \\
n_{k 1}=2, n_{k 2}=2, n_{k 3}=1 \text {. The model is estimated using the prediction error } \\
\text { method; the degree of the model selection is carried out with the best AIC } \\
\text { criterion (the structure that minimizes AIC) }\end{array}$ & FIT: $68.12 \%$, FIT1: $63.02 \%$ \\
$\begin{array}{l}\text { Black-box BJ model with } n_{b 1}=1, n_{b 2}=3, n_{b 3}=1, n_{c}=3, n_{d}=2, n_{f}=2, \\
n_{k 1}=1, n_{k 2}=3, n_{k 3}=1 . \text { The model is estimated using the prediction error } 0.138 \\
\text { method; the degree of the model selection is carried out with the best AIC } \\
\text { criterion (the structure that minimizes AIC) }\end{array}$ & FIT: $100 \%$, FIT1: $100 \%$ \\
\hline
\end{tabular}

Table 7: Milling of aluminium components

\begin{tabular}{ll}
\hline \multicolumn{1}{c}{ Parameters and polynomials } \\
\hline$B_{1}(q)=-0.00552 q^{-2}-0.006068 q^{-3}-0.003629 q^{-4}$ & $D(q)=1-1.804 q^{-1}+0.9627 q^{-2}$ \\
$B 2(q)=-0.0001954 q^{-1}$ & $F 1(q)=1+0.4775 q^{-1}+0.1816 q^{-2}$ \\
$B 3(q)=0.004336 q^{-1}$ & $F 2(q)=1-0.4527 q^{-1}+0.8147 q^{-2}$ \\
$C(q)=1-1.553 q^{-1}+0.555 q^{-2}+0.262 q^{-3}$ & $F 3(q)=1-0.554 q^{-1}+0.0992 q^{-2}$ \\
& $e(t)$ is white noise signal with variance 0.78 \\
\hline
\end{tabular}

Function and parameters that represent the behaviour of the laser-milled piece for the angle error. The degree of the BJ

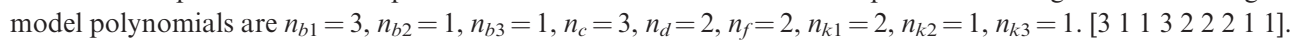

Table 8: $\quad$ Milling of aluminium components

Parameters and polynomials

\begin{tabular}{ll}
\hline$B 1(q)=-0.00909 q^{-1}$ & $D(q)=1-0.2621 q^{-1}-0.7457 q^{-2}$ \\
$B 2(q)=0.001451 q^{-3}-0.001019 q^{-4}-0.0001008 q^{-5}$ & $F 1(q)=1-0.3072 q^{-1}+0.7465 q^{-2}$ \\
$B 3(q)=-0.01077 q^{-1}$ & $F 2(q)=1-0.1005 q^{-1}+0.5109 q^{-2}$ \\
$C(q)=1+0.243 q^{-1}+0.7044 q^{-2}-0.4622 q^{-3}$ & $F 3(q)=1+0.9133 q^{-1}+0.53 q^{-2}$ \\
& $e(t)$ is white noise signal with variance 0.2
\end{tabular}

Function and parameters that represent the behaviour of the laser-milled piece for the depth error. The degree of the BJ model polynomials are $n_{b 1}=1, n_{b 2}=3, n_{b 3}=1, n_{c}=3, n_{d}=2, n_{f}=2, n_{k 1}=1, n_{k 2}=3, n_{k 3}=1 .\left[\begin{array}{llllllllllll}1 & 3 & 1 & 3 & 2 & 2 & 1 & 3 & 1\end{array}\right]$.

operation start-up costs is obtained, representing the main advantage of this proposal.

The main drawback of this process is that it cannot be completely automated because the need of experts taking part in the whole procedure as in some steps unsupervized learning is applied. Finally, it is remarkable the high precision of the obtained models as they have been found with negligible error in mostly all the cases. This is especially truth for the copper components, while for the aluminium and steel components the errors are lightly higher. It is 


Model Performance indexes

Black-box OE model with $n_{b 1}=2, n_{b 2}=1, n_{b 3}=1, n_{f}=2, n_{k 1}=1, n_{k 2}=1$, $n_{k 3}=1$. The model is estimated using the prediction error method; the degree of the model selection is carried out from the best AIC criterion (the structure that minimizes AIC)

Black-box OE model $n_{b 1}=1, n_{b 2}=1, n_{b 3}=1, n_{f}=2, n_{k 1}=1, n_{k 2}=1$, $n_{k 3}=1$. The model is estimated using the prediction error method; the degree of the model selection is carried out with the best AIC criterion (the structure that minimizes AIC)

Black-box BJ model with $n_{b 1}=1, n_{b 2}=1, n_{b 3}=1, n_{c}=2, n_{d}=2, n_{f}=2$, $n_{k 1}=1, n_{k 2}=1, n_{k 3}=1$. The model is estimated using the prediction error method; the degree of the model selection is carried out with the best AIC criterion (the structure that minimizes AIC)

Black-box BJ model with $n_{b 1}=2, n_{b 2}=1, n_{b 3}=1, n_{c}=2, n_{d}=2, n_{f}=2$, $n_{k 1}=1, n_{k 2}=1, n_{k 3}=1$. The model is estimated using the prediction error method; the degree of the model selection is carried out with the best AIC criterion (the structure that minimizes AIC)
FIT: $44.04 \%$, FIT1: $44.04 \%$

FIT10: $44.04 \%, \mathrm{~V}: 0.02$

FPE: 0.23 , NSSE: 7.71e-4

FIT: $21.2 \%$, FIT1: $21.2 \%$

FIT10: $21.2 \%$, V: 0.023

FPE: 0.162, NSSE: 0.0015

FIT: 100\%, FIT1: 100\%

FIT10: $100 \%$, V: 0.12

FPE: 0.27 , NSSE: $2.73 \mathrm{e}-31$

FIT: 100\%, FIT1: 100\%

FIT10: 100\%, V: 0.97

FPE: 1,75, NSSE: 4.17e-30

Indicator values for several proposed models of the angle error.

Table 10: $\quad$ Milling hardened steel components

Model Performance indexes

Black-box OE model with $n_{b 1}=1, n_{b 2}=2, n_{b 3}=1, n_{f}=2, n_{k 1}=1, n_{k 2}=2$, $n_{k 3}=1$. The model is estimated using the prediction error method; the degree of the model selection is carried out from the best AIC criterion FIT: $100 \%$, FIT1: $100 \%$ FIT10: $100 \%, \mathrm{~V}: 0.051$ (the structure that minimizes AIC)

Black-box BJ model with $n_{b 1}=1, n_{b 2}=3, n_{b 3}=1, n_{c}=2, n_{d}=1, n_{f}=1$, $n_{k 1}=1, n_{k 2}=2, n_{k 3}=1$. The model is estimated using the prediction error method; the degree of the model selection is carried out with the best AIC FPE: 0.636 , NSSE: $1.08 \mathrm{e}-27$ criterion (the structure that minimizes AIC)

Black-box BJ model with $n_{b 1}=2, n_{b 2}=2, n_{b 3}=2, n_{c}=2, n_{d}=1, n_{f}=1$, $n_{k 1}=2, n_{k 2}=2, n_{k 3}=1$. The model is estimated using the prediction error method; the degree of the model selection is carried out with the best AIC

FIT: $100 \%$, FIT1: 100\%

FIT10: $100 \%, \mathrm{~V}: 0.07$

FPE: 1.331 , NSSE: $1.24 \mathrm{e}-28$

FIT: $65.16 \%$, FIT1: $59.98 \%$ FIT10: $63.32 \%, \mathrm{~V}:-0.12$ FPE:0.471, NSSE:0.0014 criterion (the structure that minimizes AIC)

Indicator values for several proposed models of the depth error.

Table 11: Milling hardened steel components

Parameters and polynomials

\begin{tabular}{ll}
\hline$B 1(q)=0.01269 q^{-1}$ & $D(q)=1+1.208 q^{-1}+0.3098 q^{-2}$ \\
$B 2(q)=0.0004895 q^{-1}$ & $F 1(q)=1+0.4094 q^{-1}-0.16 q^{-2}$ \\
$B 3(q)=0.01366 q^{-1}$ & $F 2(q)=1-1.678 q^{-1}+0.7838 q^{-2}$ \\
$C(q)=1+1.541 q^{-1}+1.02 q^{-2}$ & $F 3(q)=1-1.1 q^{-1}+0.7671 q^{-2}$ \\
& $e(t)$ is white noise signal with variance 0.08
\end{tabular}

Function and parameters that represent the behaviour of the laser-milled piece for the angle error. The degree of the BJ model polynomials are $n_{b 1}=1, n_{b 2}=1, n_{b 3}=1, n_{c}=2, n_{d}=2, n_{f}=2, n_{k 1}=1, n_{k 2}=1, n_{k 3}=1 .\left[\begin{array}{llllllllll}1 & 1 & 2 & 2 & 2 & 1 & 1 & 1\end{array}\right]$. 
Table 12: $\quad$ Milling hardened steel components

Parameters and polynomials

\begin{tabular}{ll}
\hline$B 1(q)=0.003554 q^{-1}$ & $F 1(q)=1-0.4365 q^{-1}-0.1936 q^{-2}$ \\
$B 2(q)=-0.00224 q^{-2}-0.003145 q^{-3}$ & $F 2(q)=1-0.5375 q^{-1}-0.4496 q^{-2}$ \\
$B 3(q)=-0.02758 q^{-1}$ & $F 3(q)=1-1.677 q^{-1}+0.9613 q^{-2}$ \\
& $e(t)$ is white noise signal with variance 0.34
\end{tabular}

Function and parameters that represent the behaviour of the laser-milled piece for the depth error of the test piece. The degree of the OE model polynomials are $n_{b 1}=1, n_{b 2}=2, n_{b 3}=1, n_{f}=2, n_{k 1}=1, n_{k 2}=2, n_{k 3}=1$. [ $\left[\begin{array}{llllllllll}1 & 2 & 1 & 2 & 1 & 2 & 1\end{array}\right]$.
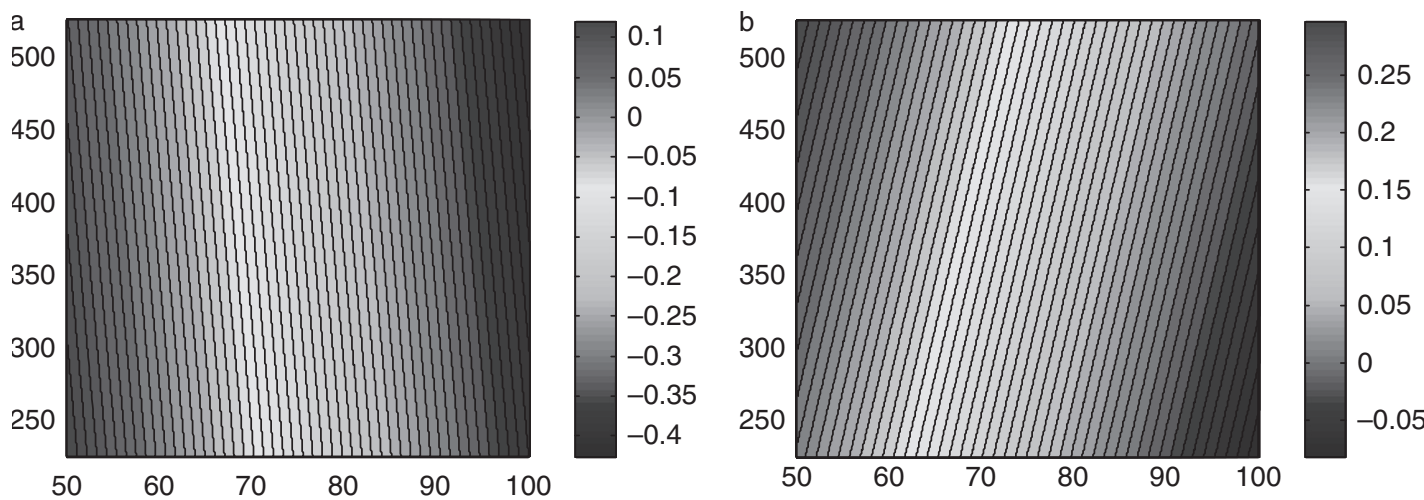

Figure 10: Output response of two different errors: the angle error ( $a$ ) and the depth error (b) for different input variable ranges.

expected that these models would be outperformed introducing different training techniques, which are left as future work.

Future work will focus on the study and application of this model to other kinds of materials of industrial interest, such as cast single-crystal nickel super-alloys for high-pressure turbine blades, and also on the application of this model to the optimization of different but similar industrial problems, such as laser cladding, laser super-polishing and laser drilling. Another interesting application and real-world, large-scale scenario is in medical therapeutics (odonto-stomatology) for bucco-dental rehabilitation and restoration in the processing and manufacturing of bucco-dental prosthesis, such as partial crowns, inlays and onlays, and partial and complete prosthesis fitted on structures of different metals, such as titanium, chrome cobalt, noble metals, etc., in which the optimizing of the registering and mapping of the surgical field to be operated on is required.
In addition, the analysis of different connectionist models will be applied for feature selection.

\section{Acknowledgements}

This research has been partially supported through the Junta de Castilla y León's project BU006A08 and the Spanish Ministry of Science and Technology's project [TIN2008-06681C06-04]. The authors would also like to thank the manufacturer of components for vehicle interiors, Grupo Antolin Ingeniería, S. A. as part of the MAGNO 2008-1028. CENIT Project funded by the Spanish Ministry of Science and Innovation.

\section{References}

Abraham, A., E. Corchado and J. Corchado (2009) Hybrid learning machines, Neurocomputing, 72, 2729-2730. 
Akaike, H. (1969) Fitting autoregressive models for prediction, Annals of the Institute of Statistical Mathematics, 20, 425-439.

Avcr, E. (2007) A new optimum feature extraction and classification method for speaker recognition: GWPNN, Expert Systems with Applications, 32, 485-498.

Avci, E., A. Sengur and D. Hanbay (2009) An optimum feature extraction method for texture classification, Expert Systems with Applications, 36, 6036-6043.

Berlanga, F., A.J. Rivera, M.J. Jesús and F. Herrera (2010) GP-COACH: genetic programming-based learning of compact and accurate fuzzy rule-based classification systems for high-dimensional problems, Information Science, 180, 1183-1200.

Bertsekas, D. (1999) Nonlinear Programming, Belmont, MA: Athena Scientific.

CALó, D.G. (2007) Gaussian mixture model classification: a projection pursuit approach, Computational Statistics and Data Analysis, 52, 471-482.

Chen, C., H. Lee and Y. Chang (2009) Two novel feature selection approaches for web page classification, Expert Systems with Applications, 36, 260-272.

Corchado, E. and C. Fyfe (2003) Connectionist techniques for the identification and suppression of interfering underlying factors, International Journal of Pattern Recognition and Artificial Intelligence, 17, 1447-1466.

Corchado, E., Y. Han and C. Fyfe (2003) Structuring global responses of local filters using lateral connections, Journal of Experimental and Theoretical Artificial Intelligence, 15, 473-487.

Corchado, E., D. MacDonald and C. Fyfe (2004) Maximum and minimum likelihood Hebbian learning for exploratory projection pursuit, Data Mining and Knowledge Discovery, 8, 203-225.

Corchado, E., P. Novais, C. Analide and J. Sedano (eds) (2010) Soft Computing Models in Industrial and Environmental Applications, 5th International Workshop (SOCO 2010), Advances in Intelligent and Soft Computing, 73, Berlin, Germany: Springer.

Cruz, C. and D. Pelta (2009) Soft computing and cooperative strategies for optimization, Applied Soft Computing, 9, 30-38.

da Silva, A., J. Neto, N. Nagem and N. Freixo (2009) Parametric ARX modeling of the electrolytic smelter pot, in UKSIM '09: Proceedings of the UKSim 2009: 11th International Conference on Computer Modelling and Simulation, E. Coll (ed.), Washington, DC: IEEE Computer Society, 217-222.

Das, S., A. Abraham and A. Konar (2008) Automatic kernel clustering with a multi-elitist particle swarm optimization algorithm, Pattern Recognition Letters, 29, 688-699.

Datong, L., P. Yu and P. Xiyuan (2009) Online fault prediction based on combined AOSVR and ARMA models, in Testing and Diagnosis, 2009. ICTD 2009
IEEE Circuits and Systems International Conference, New York, USA, pp. 1-4.

Diaconis, P. and D. Freedman (1984) Asymptotics of graphical projections, Annals of Statistics, 12, 793-815.

Esbensen, K. and P. Geladi (2009) Principal component analysis: concept, geometrical interpretation, mathematical background, algorithms, history, practice, in Comprehensive Chemometrics, S.D. Brown, R. Tauler and B. Walczak (eds), Oxford: Elsevier, 211-226.

Fernandes, C., P. Comon and G. Favier (2010) Blind identification of MISO-FIR channels, Signal Processing, 90, 490-503.

FöldiÁK, P. (1992) Models of sensory coding, PhD Thesis, University of Cambridge.

Friedman, J. and J. Tukey (1974) Projection pursuit algorithm for exploratory data-analysis, IEEE Transactions on Computers, 23, 881-890.

Fyfe, C. (1993) PCA properties of interneurons: from neurobiology to real world computing, in International Conference on Artificial Neural Networks, S. Gielen and B. Kappen (eds), Berlin, Germany: Springer Verlag, 183-188.

Gavrilis, D., I.G. Tsoulos and E. Dermatas (2008) Selecting and constructing features using grammatical evolution, Pattern Recognition Letters, 29, 1358-1365.

GillberG, J. and L. Ljung (2010) Frequency domain identification of continuous-time output error models, part II: non-uniformly sampled data and Bspline output approximation, Automatica, 46, 11-18.

Gonzalez, F., L. Munoz and A. Belanche (2009) Gene subset selection in microarray data using entropic filtering for cancer classification, Expert Systems, 26, 113-124.

Guyon, I. and A. ElisseefF (2003) An introduction to variable and feature selection, Journal of Machine Learning Research, Special Issue on Variable and Feature Selection, 3, 1157-1182.

Haber, R. and L. Keviczky (1999a) Nonlinear System Identification. Input-Output Modeling Approach. Part 1: Nonlinear System Parameter Estimation, Dordrecht, MA: Kluwer Academic Publishers.

Haber, R. and L. KeviczKy (1999b) Nonlinear System Identification. Input-Output Modeling Approach. Part 2: Nonlinear System Structure Identification, Dordrecht, MA: Kluwer Academic Publishers.

Harrison, P.M., M. Paul, M. Henry, I. Henderson and M. Brownell (2004) Laser milling of metallic and nonmetallic substrates in the nanosecond regime with Q-switched diode pumped solid state lasers, in High-Power Laser Ablation V. Proceedings of the Society of Photo-optical Instrumentation Engineers: SPIE, Vol. 5448, pp. 624-633.

Henry, M., P. Harrison, I. Henderson and M. BrowNELL (2004) Laser milling - a practical industrial 
solution for machining a wide variety of materials, in 5th International Symposium on Laser Precision Microfabrication. Proceedings of the Society of Photooptical Instrumentation Engineers, Nara, Japan: SPIE, Vol. 5662, pp. 627-632.

Herrero, A., E. Corchado, L. Sáiz and A. Abraham (2010) DIPKIP: a connectionist knowledge management system to identify knowledge deficits in practical cases, Computational Intelligence, 26, 26-56.

Hotelling, H. (1933) Analysis of a complex of statistical variables into principal components, Journal of Education Psychology, 24, 417-444.

Huang, J., H. Luo, B. Long and H. Wang (2009) Prediction research about small sample failure data based on ARMA model, in Testing and Diagnosis, 2009. ICTD 2009. IEEE Circuits and Systems International Conference on, New York, USA, pp. 1-6.

Ion, J. (2005) Laser Processing of Engineering Materials: Principles, Procedure and Industrial Application, Oxford, UK: Butterworth-Heinemann, 456-475.

Iqbal, M., U. Sundararaj and S. Shah (2010) New approach to develop dynamic gray box model for a plasticating twin-screw extruder, Industrial and Engineering Chemistry Research, 49, 648-657.

Ismail, N., N. TaJuUdin, M. Rahiman and M. TAib (2009) Modeling of dynamic response of essential oil extraction process, Proceedings of the 5th International Colloquium on Signal Processing and its Applications (CSPA), 6-8 March 2009, Kuala Lumpur, Malaysia, pp. 298-301.

KIM, K.J. and S.B. Сно (2006) Ensemble classifiers based on correlation analysis for DNA microarray classification, Neurocomputing, 70, 187-199.

Kohonen, T. (2006) Self-organizing neural projections, Neural Networks, 19, 723-733.

KunL, M. (2002) From macro to micro - the development of laser ablation, in International Congress on Applications of Lasers \& Electro Optics (ICALEO), Scottsdale, Arizona, USA.

Liu, H., J. Sun, L. Liu and H. Zhang (2009a) Feature selection with dynamic mutual information, Pattern Recognition, 42, 1330-1339.

LiU, H. and L. Yu (2005) Toward integrating feature selection algorithms for classification and clustering, IEEE Educational Activities Department, 17, 491-502.

Liv, Y., Y. LiU and K.C. ChAN (2009b) Dimensionality reduction for heterogeneous dataset in rushes editing, Pattern Recognition, 42, 229-242.

Luung, L. (1999) System Identification. Theory for the User, 2nd edn, Upper Saddle River, NJ: Prentice-Hall.

Lorena, A. and A.C.P.F. DE Carvalho (2008) Evolutionary design of code-matrices for multiclass problems, in Soft Computing for Knowledge Discovery and Data Mining, O. Maimon and L. Rokach (eds), New York, NY: Springer, 153-184.
Malpica, J.A., J.G. Rejas and M.C. Alonso (2008) A projection pursuit algorithm for anomaly detection in hyperspectral imagery, Pattern Recognition, 41, 3313-3327.

Marinakis, Y., M. Marinaki, G. Dounias, J. Jantzen and B. BJERREGAARD (2009) Intelligent and nature inspired optimization methods in medicine: the Pap smear cell classification problem, Expert Systems, 26, 433-457.

Meiler, A., O. Schimida, M. Schudy and E. Hofer (2008) Dynamic fuel cell stack model for real-time simulation based on system identification, Journal of Power Sources, 176, 523-528.

Mostafa, M. and N. Billor (2009) Recognition of western style musical genres using machine learning techniques, Expert Systems with Applications, 36, 11378-11389.

Mustafaraj, G., J. Chen and G. Lowry (2010) Development of room temperature and relative humidity linear parametric models for an open office using BMS data, Energy and Buildings, 42, 348-356.

Naldi, M., A.C.P.F. de Carvalho, R. Gabrielli and E. HruschKa (2008) Genetic clustering for data mining, in Soft Computing for Knowledge Discovery and Data Mining, Vol. 2, O. Maimon and L. Rokach (eds), New York, NY: Springer, 113-132.

Nelles, O. (2001) Nonlinear System Identification. From Classical Approaches to Neural Networks and Fuzzy Models, Berlin, Germany: Springer.

NørgaArd, M., O. Ravn, N. Poulsen and L. HANSEN (2000) Neural Networks for Modelling and Control of Dynamic Systems, London UK: SpringerVerlag.

OJA, E. (1982) A simplified neuron model as a principal component analyzer, Journal of Mathematical Biology, 15, 267-273.

Pearson, K. (1901) On lines and planes of closest fit to systems of points in space, Philosophical Magazine, 2, 559-572.

Raducanu, B., Ã.J. Vitri and A. Leonardis (2010) Online pattern recognition and machine learning techniques for computer-vision: theory and applications, Image and Vision Computing, 28, 1063-1064.

Seung, H., N. Socci and D. Lee (1998) The rectified Gaussian distribution, Advances in Neural Information Processing Systems, 10, 350-356.

Söderström, T. and P. Stoica (1989) System Identification, Englewood Cliffs, NJ: Prentice Hall International.

Su, T.H., T.W. Zhang, D.J. Guan and H.J. Huang (2009) Off-line recognition of realistic Chinese handwriting using segmentation-free strategy, Pattern Recognition, 42, 167-182.

TAghavi, N. and A. SAdR (2008) Piezoelectric transducer modeling: with system identification (SI) method, in Proceedings of World Academy of Science, Engineering and Technology, Bangkok, Thailand, Vol. 29, pp. 296-301. 
Tani, G., L. Orazi, A. Fortunato and G. Cuccolini (2008) Laser ablation of metals: a 3D process simulation for industrial applications, Journal of Manufacturing Science and Engineering, 130, 31111-31122.

Torra, V. and Y. NARUKAWA (2010) Soft computing in decision modeling, Soft Computing - A Fusion of Foundations, Methodologies and Applications, 14, 419-420.

Valeriana-Garcia, R., J. Fernandez-Breis, J. RuizMartinez, F. Garcia-Sanchez and R. MartinezBeJAR (2008) A knowledge acquisition methodology to ontology construction for information retrieval from medical documents, Expert Systems, 25, 314-334.

Wang, R.Q. and W.J. Cheng (2009) Using ARmaXEGARCH model to forecast day-ahead electricity prices for PJM market, flushing, Proceedings of International Conference of Management Science and Information System, 28-30 September 2009, Jiaozuo City, China, 1-4, pp. 1140-1144.

Witte, R., T. Moser, R. Liebers and R. Holtz (2008) Laser micro-drilling with nanoseconds: parametrical influences and results, in : Advanced Laser Technologies. Proceedings of the Photo-Optical Instrumentation Engineering, Conference on Advanced Laser Technologies, Levi, Finland, J Manuf (ed.), SPIE, 7022, 702208-702216.

Wolczowski, A. and M. Kurzynski (2010) Humanmachine interface in bioprosthesis control using EMG signal classification, Expert Systems, 27, 53-70.

Wright, J., A. YANG and A. GANesh (2009) Robust face recognition via sparse representation, IEEE Transactions on Pattern Analysis and Machine Intelligence, 31, 210-227.

ZADEH, L. (2009) Toward extended fuzzy logic - a first step, Fuzzy Sets and Systems, 160, 3175-3181.

\section{The authors}

\section{Emilio Corchado}

Emilio Corchado is an associated professor at the University of Salamanca (Spain). He received his $\mathrm{PhD}$ in computer science and his degree in physics from University of Salamanca (Spain). His research interests include neural networks, with a particular focus on exploratory projection pursuit, maximum likelihood Hebbian learning, self-organising maps, multiple classifier systems and hybrid systems. He has published over 80 peer-reviewed articles in a range of topics from knowledge management and risk analysis, intrusion detection systems, food industry, artificial vision and recently modelling of industrial processes. $\mathrm{He}$ is the director of the GICAP Research Group at the University of Burgos, Spain. He is coeditor-inchief of International Journal of Computational Intelligence Research (IJCIR) and member of the editorial boards of the International Journal of Computational Intelligence and Applications (IJCIA) and also of the International Journal of Reasoning-based Intelligent Systems (IJRIS). He is the director of HAIS international series of conferences and codirector of CISIS international series of conferences.

\section{Javier Sedano}

Javier Sedano obtained his $\mathrm{PhD}$ at the University of León, degree in industrial engineering. He has participated in the development and management of more than 25 technological development projects and pre-funded in regional and national programs, and prototypes most cases. Training of professionals in all disciplines of production technologies and project management. He has published articles in international prestige, as well as over 25 contributions, including book chapters and conference communications. $\mathrm{He}$ is the author of intellectual property records, which are in operation.

\section{Leticia Curiel}

Leticia Curiel is an assistant lecturer at the University if Burgos. She obtained the computer sciences degree at the University of Leon (Spain) and the $\mathrm{PhD}$ in computer sciences at University of Burgos (Spain). She has published in international journals and conferences. She has been member of the program committee of some conference like International Conference on Intelligent Data Engineering and Automated Learning (IDEAL), International Conference on Hybrid Artificial Intelligence Systems (HAIS), International Workshop on Computational Intelligence in Security for Information Systems (CISIS), International Workshop on Soft Computing Models in Industrial Applications 
(SOCO) and International Workshop on Practical Applications of Agents and Multiagent Systems. (IWPAAMS).

\section{José R. Villar}

José R. Villar born on 11 October 1967 in Santo Domingo, Dominican Republic. He obtained the engineering degree at the University of Oviedo and the $\mathrm{PhD}$ in computer sciences at University of León, both in Spain. He had worked with several engineering consultancies. He began his educational career at University of León. Currently he is with the computer science Department at University of Oviedo. He is the author of several articles published in different international and indexed journals, more than 50 international conference contributions, three patents and more than ten research projects funded by national grants or private investment. 